\title{
RELACÃO ENTRE IDADE, FUNCIONALIDADE E FORÇA DE PREENSÃO MANUAL EM IDOSOS INSTITUCIONALIZADOS
}

\author{
Leonardo Squinello Nogueira Veneziano' \\ Jéssica Silva do Nascimento ${ }^{2}$ \\ Nulciene Firmino de Freitas ${ }^{3}$ \\ Getúlio Antonio de Freitas Filho ${ }^{4}$ \\ Fernando Duarte Cabral ${ }^{5}$ \\ Roberto Dias $^{6}$ \\ Lya Karla Manso Miranda ${ }^{7}$ \\ Kátia Silveira Ferreira ${ }^{8}$ \\ Renata do Nascimento Silva ${ }^{9}$ \\ Tairo Vieira Ferreira ${ }^{10}$ \\ Rejane Maria Cruvinel Cabral ${ }^{11}$
}

Resumo: Os idosos apresentam características particulares, dentre as quais se destaca a diminuição da força muscular. A dinamometria manual tem sido utilizada em diversos campos da área da saúde, consiste em um teste simples, confiável e objetivo de aferição da força máxima voluntária de preensão manual. $O$ objetivo do presente estudo foi estabelecer a associação entre a idade, a funcionalidade e a força de preensão manual dos idosos. Trata-se de um estudo cuja amostra foi composta por 35 idosos do sexo masculino, com idades entre 60 e 90 anos. Para avaliação da força de preensão manual foi utilizado o Dinamômetro Hidráulico Manual da marca Crown. A avaliação da funcionalidade foi feita através do índice de Katz, que avalia seis tarefas do dia a dia. Idosos entre 60 e 69 anos de idade obtiveram uma força média de preensão de 15,38kg/f, idosos de 70 a 79 anos apresentaram força média de 11,4kg/f, e os idosos de 80 a 90 anos tem força média de 8,6kg/f. pode-se concluir que a força de preensão manual apresentou maior declínio quando relacionada à idade, ou seja, quanto maior a idade menor a força e maior dependência funcional.

Palavras-chave: Institucionalização; Idosos; Funcionalidade; Força de preensão manual.

\footnotetext{
1 Instituto de Ensino Superior de Rio Verde, Brasil. E-mail: leosnv@yahoo.com.br.

2 Instituto de Ensino Superior de Rio Verde, Brasil. E-mail: jessica@faculdadeobjetivo.com.br.

${ }^{3}$ Instituto de Ensino Superior de Rio Verde, Brasil. E-mail: nulciene@faculdadeobjetivo.com.br

4 Instituto de Ensino Superior de Rio Verde, Brasil. E-mail: getulio@faculdadeobjetivo.com.br.

${ }^{5}$ Instituto de Ensino Superior de Rio Verde, Brasil. E-mail: fernando@faculdadeobjetivo.com.br.

6 Instituto de Ensino Superior de Rio Verde, Brasil. E-mail: fisiodias@uol.com.br.

7 Instituto de Ensino Superior de Rio Verde, Brasil. E-mail: lya@faculdadeobjetivo.com.br.

8 Instituto de Ensino Superior de Rio Verde, Brasil. E-mail: katia@faculdadeobjetivo.com.br.

9 Instituto de Ensino Superior de Rio Verde, Brasil. E-mail: renata@faculdadeobjetivo.com.br.

10 Instituto de Ensino Superior de Rio Verde, Brasil. E-mail: tairo@faculdadeobjetivo.com.br.

11 Instituto de Ensino Superior de Rio Verde, Brasil. E-mail: rejane@faculdadeobjetivo.com.br.
} 